\title{
health and safety laboratory
}

LOW-ENERGY ATMOSPHERIC GAMMA RADIATION AS A MEASURE OF COSMIC-RAY SECONDARY ELECTRON FLUX

Wayne M. Lowder June 1969

\section{UNITED STATES ATOMIC ENERGY COMMISSION}


This report was prepared as an account of Government sponsored work. Neither the United States, nor the Commission, nor any person acting on behalf of the Commission:

A. Makes any warranty or representation, expressed or implied, with respect to the accuracy, completeness, or usefulness of the information contained in this report, or that the use of any information, apparatus, method, or process disclosed in this report may not infringe privately owned rights; or

B. Assumes any liabilities with respect to the use of, or for damages resulting from the use of any information, apparatus, method, or process disclosed in this report.

As used in the above, "person acting on behalf of the commission" includes any employee or contractor of the Commission, or employee of such contractor, to the extent that such employee or contractor of the Commission, or employee of such contractor prepares, disseminates, or provides access to, any information pursuant to his employment or contract with the Commission, or his employment with such contractor.

Printed in the United States of America Available from

clearinghouse for Federal Scientific and Technical Information National Bureau of Standards, U. S. Department of Commerce Springfield, Virginia 22151

Price: Printed Copy $\$ 3.00$; Microfiche $\$ 0.65$ 


\section{DISCLAIMER}

This report was prepared as an account of work sponsored by an agency of the United States Government. Neither the United States Government nor any agency Thereof, nor any of their employees, makes any warranty, express or implied, or assumes any legal liability or responsibility for the accuracy, completeness, or usefulness of any information, apparatus, product, or process disclosed, or represents that its use would not infringe privately owned rights. Reference herein to any specific commercial product, process, or service by trade name, trademark, manufacturer, or otherwise does not necessarily constitute or imply its endorsement, recommendation, or favoring by the United States Government or any agency thereof. The views and opinions of authors expressed herein do not necessarily state or reflect those of the United States Government or any agency thereof. 


\section{DISCLAIMER}

Portions of this document may be illegible in electronic image products. Images are produced from the best available original document. 


\title{
LOW-ENERGY ATMOSPHERIC GAMMA RADIATION AS A MEASURE OF COSMIC-RAY SECONDARY ELECTRON FLUX
}

\author{
Wayne M. Lowder
}

June 1969

Health and Safety Laboratory

U. S. Atomic Energy Commission New York, New York

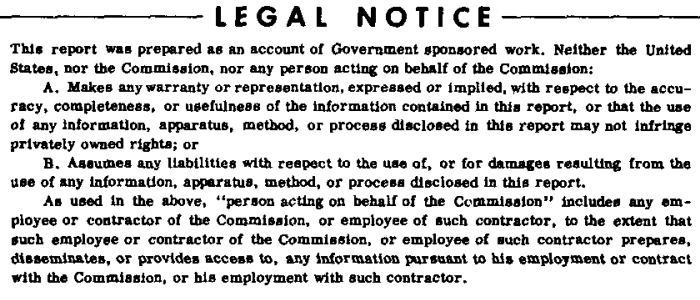




\section{ABSTRACT}

A method is described for determining the secondary electron flux from low-energy gamma-ray measurements which will be useful in our investigations of the properties of the cosmic radiation field within the atmosphere. A quantitative relation between the omnidirectional positron (and total electron) flux and $0.51 \mathrm{MeV}$ annihilation photon flux is derived, applicable to atmospheric depths $>50 \mathrm{~g} / \mathrm{cm}^{2}$. This relation can be used to calibrate any experimental measure of low-energy photons, produced primarily by electron bremsstrahlung, in terms of total electron flux. Gamma-ray data from two balloon flights have been used to determine an absolute electron flux profile throughout the atmosphere that is consistent with existing experimental data. 
TABLE OF CONTENTS

TEXT

Page

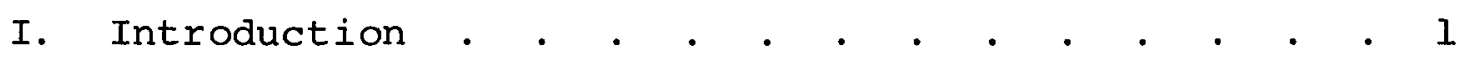

II. Production and Transport of Cosmic-Ray Positrons - 3

A. Definition of Positron "Absorption"

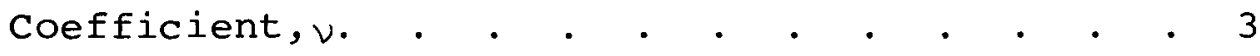

B. Determination of $\nu$ for Atmospheric

Positrons. . . . . . . . . . . . . 6

C. Relation of Positron Flux to Source • • • . 8

III. Absorption of Cosmic-Ray Positrons and Production of $0.51 \mathrm{MeV}$ Gamma-Rays . . . . . . . . . . 10
A. Production Efficiency of $0.51 \mathrm{MeV}$ Photons per Positron Annihilation. . . . . . . . 10
B. Relation of $0.51 \mathrm{MeV}$ Gamma Source to Positron Source • . . . . . . . . . . 12
C. Relation of $0.51 \mathrm{MeV}$ Photon Flux to

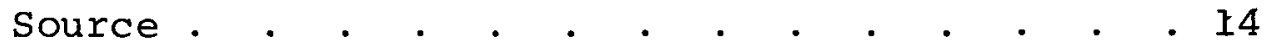

IV. Relation Between Electron and $0.51 \mathrm{MeV}$ Photon Fluxes in the Atmosphere . . . . . . . . . 15
A. Positron Flux as a Function of $0.51 \mathrm{MeV}$
Photon Flux . • • . • . • • • • • . 15
B. Charge Ratio of Cosmic-Ray Secondary Electrons. 15
C. Final Result . . . . . . . . . . . . . . 16 
TABLE OF CONTENTS (Cont'd)

Page

V. Determination of Electron Flux from Gamma-Ray

Measurements . . . . . . . . . . . . . 17

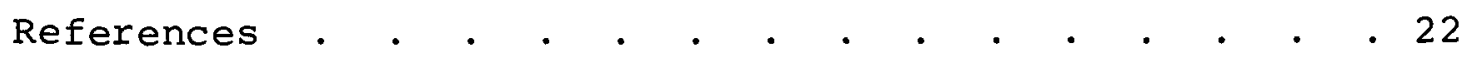

TABLE

Table I. Experimental and Inferred Photon and Particle

Fluxes . . . . . . . . . . . . . 25

APPENDICES

Appendix I. Calculation of Positron Energy Spectrum

at Production. . . . . . . . . . 26

Appendix II. Calculation of Positron Flux in the

Atmosphere . . . . . . . . . . . 28

Appendix III. Calculation of $0.51 \mathrm{MeV}$ Gamma-Ray Flux in the Atmosphere. . . . . . . . . 31

\section{ILLUSTRATIONS}

Figure 1. Integral electron energy spectrum in the atmosphere $\left(\mathrm{h}>70 \mathrm{gm} / \mathrm{cm}^{2}\right) . .5 . .33$

Figure 2. Integral positron range spectrum in the atmosphere $\left(\mathrm{h}>70 \mathrm{gm} / \mathrm{cm}^{2}\right)$. . . . . 34

Figure 3. Secondary electron flux vs. atmospheric depth, Palestine, Texas, 1967 . . . . 35 


\section{INTRODUCTION}

Direct experimental investigations of the propagation of the various primary and secondary components of the cosmic radiation through the atmosphere have been seriously limited by difficulties in discriminating among them. These difficulties pertain particularly to omnidirectional charged particle detectors, whose geometry is unsuitable for the application of most of the common experimental techniques providing such discrimination. Our primary interest is the determination of the total ionization and dose rate in the atmosphere from the total and component radiations at all altitudes, which requires an improved understanding of the response to cosmic radiation of standard omnidirectional detectors, particularly the ionization chamber. (1) For such applications, the relevant experimental data are limited to (I) the relative responses of particular omnidirectional detectors surrounded by various thicknesses of absorber, (2) the relative responses of several detectors with different response characteristics to the various components, and (3) measurements over a large range of angles with directional detectors. However, the data from such experiments are sparse and the problems of interpretation substantial.

In this report, we discuss a method by which the electron component of the omnidirectional charged particle flux can be uniquely distinguished from the total. The electrons constitute a significant fraction of this total flux at almost all altitudes, and thus an independent determination of the intensity of this component would considerably simplify the analysis of the total radiation field.

This method is based on the fact that, in the absence of significant quantities of gamma-ray-emitting radioisotopes, the presence of a discrete line in the atmospheric gamma-ray energy spectrum at $0.51 \mathrm{MeV}$ can be uniquely related to the annihilation of low-energy $\left(E_{k i n}<<m_{e} c^{2}\right)$ 
cosmic-ray positrons. The source density of $0.51 \mathrm{MeV}$ photons can be calculated from the observed flux, and related to the absorption density of positrons. We find that the linear range spectrum of positrons produced can be inferred from the properties of the positron flux, and that this spectrum can be approximated by an exponential function. We can then treat the positrons as pseudophotons in terms of effective absorption, and quantitatively relate the positron and $0.51 \mathrm{MeV}$ photon fluxes in a relatively simple manner.

The derivation of the equation relating the $0.51 \mathrm{MeV}$ gamma-ray flux to the total electron flux involves prior consideration of the production, transport, and absorption of cosmic-ray positrons and $0.51 \mathrm{MeV}$ gamma-rays. We require quantitative estimates of the energy and range spectrum of the positrons produced (Section II) and the efficiency of "conversion" of annihilating positrons to $0.51 \mathrm{MeV}$ photons (Section III), as well as the functional relationships relating positron production to total electron flux and $0.51 \mathrm{MeV}$ photon production to the measured photon flux (Section IV).

The $0.51 \mathrm{MeV}$ gamma-ray flux cannot be readily measured at all altitudes, and thus cannot be used to infer directly the altitude variation of electron flux throughout the atmosphere. In section $V$, we shall discuss the use of total gamma-ray measurements for determining this profile. 


\section{PRODUCTION AND TRANSPORT OF COSMIC-RAY POSITRONS}

\section{A. Definition of Positron "Absorption" Coefficient, $\nu$}

Any quantitative relationship between the measured 0.51 $\mathrm{MeV}$ photon flux and the inferred positron flux at any point will necessarily contain parameters describing the transport of these particles from production to effective absorption. We seek a quantitative expression for the "Iinear range" spectrum of positrons produced in the atmosphere, $S_{p}(R, h)$, where the linear range $R$ is the thickness of air in $\mathrm{gm} / \mathrm{cm}^{3}$ between the point of production and point of annihilation and $h$ is the atmospheric depth in $\mathrm{gm} / \mathrm{cm}^{2}$.

If an exponential function can be fitted to this spectrum, we can then define a positron "absorption" coefficient, $\nu$, that is analogous for our purposes to the photon absorption coefficient, $\mu$.

The starting point of our investigation is the energy spectrum of the total electron* flux as a function of atmospheric depth. This energy spectrum at any depth can be converted to an integral range spectrum by making use of the diffusion model of high-energy electron transport to calculate the probability $\eta\left(E_{0}, Z, R\right)$ that an electron of initial energy $E_{0}$ will penetrate at least a Iinear distance $\mathrm{R}\left(\mathrm{gm} / \mathrm{cm}^{2}\right)$ through, a material of atomic number $\mathrm{Z}$, i.e.,

$$
\eta\left(E_{0}, Z, R\right)=I-\Phi\left(5.03 R / L-3.83 \lambda_{0} / I-0.84\right) \text { (II-I) }
$$

where

$$
\begin{aligned}
& \Phi(\mathrm{x})=(1 / \sqrt{\pi}) \int_{-\infty}^{\mathrm{x}} \exp \left(-\mathrm{t}^{2}\right) \mathrm{dt} \\
& \lambda_{0} / \mathrm{L}=\left(1+0.76 \mathrm{z} \mathrm{mec}^{2} / \mathrm{E}_{0}\right)^{-1}
\end{aligned}
$$

*To reduce ambiguity, the term "negatron" is used in this paper to refer specifically to negatively-charged electrons, and the term "electron" reserved for the total component, irrespective of the sign of the charge. 
and $L$ is the mean path length ("range") according to the tables of Berger and Seltzer. ( $z$ ) Harder and Poschet ( 3 ) have experimentally verified to within $5 \%$ the validity of equation (II-l) for $\mathrm{E}_{0}<30 \mathrm{MeV}$ in carbon, aluminum, copper, and lead. Assuming the validity of this equation at higher energies in air, the integral range spectrum is then inferred by means of the equation

$$
\int_{R_{0}}^{\infty} \varphi_{e}(R) d R=\int_{0}^{\infty} \varphi_{e}(E) \eta\left(E, R_{0}\right) d E
$$

where $\varphi_{e}(E)$ is the differential energy spectrum of the electron flux and $\varphi_{e}(R)$ is the corresponding differential range spectrum.

We now proceed to make the assumption that the integral range spectrum of the positron flux is identical to that calculated for the total electron flux. Although this assumption effectively ignores those processes in the production and transport of positrons and negatrons that tend to harden the positron flux spectrum relative to that of the negatrons (annihilation in flight, production of negatrons by compton scattering, differences in energy loss), the error introduced is not significant relative to the uncertainties in the preceding calculation of the electron range spectrum.

The final step in this analysis anticipates the fact that this positron flux integral range spectrum can be adequately approximated by an exponential function, i.e., that

$$
\int_{R_{0}}^{\infty} \varphi_{p}(R, h) d R \cong \Phi_{p}(h) e^{-\nu R_{0}}
$$

For it is clear that the following relation between the flux range spectrum $\varphi_{p}(R, h)$ and the source range spectrum $\mathrm{s}_{\mathrm{p}}(\mathrm{R}, \mathrm{h})$ also holds 
$\int_{R_{0}}^{\infty} \varphi_{p}(R, h) d R=\int f(r) \rho\left(h^{\prime}\right)\left[\int_{t+R_{0}}^{\infty} s_{p}\left(R, h^{\prime}\right) d R\right] d V$

where $f(r)$ is equal to $1 / 4 \pi r^{2}$ for isotropic emission of positrons and to unity for emission in one direction, and $t$ is the radius vector (in $\mathrm{gm} / \mathrm{cm}^{2}$ ) from source to flux detector at depth $h_{\text {.* }}$ Equating the right sides of equations (3) and (4), and remembering that $t$ and $R_{0}$ are arbitrary, we get

$$
S_{p}(R, h) \cong \nu e^{-\nu R} \int_{0}^{\infty} S_{p}(R, h) d R
$$

It follows that

$$
\int_{R_{0}}^{\infty} S_{p}(R, h) d R \cong e^{-\nu R_{0}} \int_{0}^{\infty} S_{p}(R, h) d R
$$

This is of the same form as equation (3), and thus the source integral range spectrum is of the same form as that for the flux. This permits us to obtain a value for $\nu$ applicable to the positron source directly from the integral range spectrum of the flux.

The situation described by equations (3) and (6) is analogous to that for photons, where the same total attenuation coefficient, $\mu$, describes the effective range distribution of both the photon source and the flux of primary photons at any point. This is relevant to our present analysis since we measure the primary $(0.51 \mathrm{MeV})$ photon flux from positron annihilation, and any interaction removes a particular photon from the discrete peak in the gamma-ray spectrum at $0.51 \mathrm{MeV}$. It is evident that, in these circumstances, the functional relationship

*A complete definition of symbols is given in Appendix II. 
between the positron and annihilation gamma-ray fluxes depends in a straightforward manner on $\mu$ and $\nu$, as well as on the mean number of $0.51 \mathrm{MeV}$ gamma-rays produced per positron absorbed (Section III) and the parameters describing the spatial distribution of the positron source (Section IV).

\section{B. Determination of $\nu$ for Atmospheric Positrons}

The most detailed information on the omnidirectional cosmic-ray electron energy distribution in the atmosphere is given by Carmichael, ( 4$)$ who determined near sea-level the pulse height spectrum produced by ionizing particles penetrating various thicknesses of shielding surrounding a pressurized argon ionization chamber. He combined the derived integral energy spectrum for the electron flux above $136 \mathrm{MeV}$ with the best available data at lower energies from earlier investigations $(5, \theta)$ and a measure of the total flux above $0.7 \mathrm{MeV}$ to produce the integral spectrum shown in Figure 1.

We now consider how the shape of this spectrum may vary with atmospheric depth. Barker's data(B) indicate that there is no appreciable change in the spectrum between 600 and $1000 \mathrm{gm} / \mathrm{cm}^{2}$ depth. The calculation of the vertical electron fluxes of energies $>0,>6$, and $>26 \mathrm{MeV}$ at depths less than $500 \mathrm{gm} / \mathrm{cm}^{2}$ by Charakhchian and Charakhchian $(7)$ indicates that the integral spectra are nearly identical in form below $\sim 100 \mathrm{gm} / \mathrm{cm}^{2}$, and in rough agreement with the sea-level spectrum in Figure 1. At lesser depths, the spectrum hardens considerably, although the omnidirectional flux spectrum would show less significant hardening with increasing altitude than the vertical spectrum.

This apparent near-constancy of the atmospheric electron spectrum can be qualitatively understood by considering the production mechanism of free electrons. Four processes are operative in such production: (1) gamma-ray interactions (pair production and compton scattering), (2) muon decay, 
(3) beta decay of radioisotopes produced by nucleon interactions with nitrogen and oxygen, and (4) "knock-on" collisions of charged particles with nitrogen and oxygen. We need not consider each of these processes in detail since (1) and (2) are dominant at all altitudes, and the overwhelming majority of electrons in the atmosphere are produced in electromagnetic cascade showers initiated by photons from neutral pion decay and by electrons from muon decay (see, e.g., Rocchia( $(8)$ ). The energy distribution of electrons in such showers is a relatively insensitive function of the nature of the initiating event and the depth of penetration of the shower. The air cascade calculations of Butcher and $\operatorname{Messel}^{(\theta)}$ and $\operatorname{Beck}^{(10)}$ indicate that the sea-level spectrum in Figure 1 is closely matched by electron flux spectra in cascades initiated by electrons and photons of 500-1000 MeV after development through more than $100 \mathrm{gm} / \mathrm{cm}^{2}$ of air. The explanation of the apparent constancy in the cosmic-ray electron spectrum at depths where the absence of shower-producing events above the top of the atmosphere has Iittle effect may lie in the fact that middle-aged cascades from moderately high energy initiating particles are the most important contributor to the observed flux. Near the top of the atmosphere, the average age of the showers rapidly decreases with increasing altitude, and the electron spectrum hardens.

These considerations lead us to make the provisional assumption that the omnidirectional electron spectrum at altitudes below the pfotzer maximum is adequately described by the sea-level spectrum in Figure 1 . The validity of this assumption will be further supported by comparisons of the results derived from it with additional experimental and analytical data.

From the energy spectrum of the electron flux in Figure 1 we obtain the linear range spectrum for the positron flux shown in Figure 2 by the method described in Section IIA (equation II-2). Also plotted in Figure 2 is the function $\exp (-0.085 \mathrm{R})$, which approximates to within \pm $10 \%$ the range aistribution of the positron flux for $280 \%$ of 
the positrons $\left(\mathrm{R}<20 \mathrm{gm} / \mathrm{cm}^{2}\right)$. The calculated range spectrum in Figure 2 for $R>20 \mathrm{gm} / \mathrm{cm}^{2}$ may be somewhat in error, because the numerical integration of equation II-2 did not take into account the details of the energy spectrum above $120 \mathrm{MeV}$ and the process of positron annihilation in flight.

It is noteworthy that the absorption data of charakhchian and Charakhchian $(\gamma)$ for electrons of $<30 \mathrm{MeV}$ at depths between 100 and $500 \mathrm{gm} / \mathrm{cm}^{2}$ at $51^{\circ} \mathrm{N}$ latitude, indicated in Figure 2, are quite consistent with the calculated range spectrum. This further supports the assumptions made in the derivation of this spectrum.

Since the indicated exponential function in Figure 2 provides an adequate approximation to the positron flux range spectrum, equation II-6 holds, where $\nu \cong 0.085 \mathrm{~cm}^{2} / \mathrm{gm}$. This form for the positron production spectrum is consistent with the Monte carlo calculations of $\operatorname{Beck}_{9}^{(10)}$ described in Appendix I.

\section{Relation of Positron Flux to Source}

We have now established that at a particular depth, $h$, the emitted positrons have an integral range spectrum described by equation II-6 with $\nu=0.085 \mathrm{~cm}^{2} / \mathrm{gm}$. This permits a calculation of the relation of the positron flux to the source once the spatial dependence of positron production is given. This spatial dependence can be estimated from the observation that experimental measures of charged particle fluxes $(13.13)$ in the atmosphere can be approximated by exponential functions over depth ranges much larger than $1 / v$, with effective attenuation lengths, $\alpha$, such that $\alpha<.01 \ll \nu$. Since the angular distribution of emitted positrons should not be a sensitive function of atmospheric depth, this exponential variation of the flux can only be explained as a reflection of the same variation in the source. In Appendix II. we introduce this exponential variation of the positron source function (equation 5) into the derivation of the functional relationship between positron flux and source density, which yields the expression 


$$
\Phi_{p}(h) \cong s_{p}(h) / \nu
$$

under the conditions that $\nu \mathrm{h} \gg \mid$ and $\alpha \ll \nu$. The abovementioned experimental data indicate that the latter condition is satisfied throughout the atmosphere, while the former condition is satisfied for those depths where equation II-6 holds, i.e., $\mathrm{h} \geq 70 \mathrm{gm} / \mathrm{cm}^{2}$. 


\section{ABSORPTION OF COSMIC-RAY POSITRONS AND PRODUCTION}

OF $0.51 \mathrm{MeV}$ GAMMA-RAYS

A. Production Efficiency of 0.51 - MeV Photons per Positron Annihilation

We now consider the relation between the absorption of cosmic-ray positrons and the appearance of a discrete gammaray flux at $0.51 \mathrm{MeV}$. All positrons are eventually subject to the process of annihilation, but such positrons produce a pair of $0.511-\mathrm{MeV}$ photons only when unbound at near-thermal kinetic energies or when bound in the singlet state of positronium. When a positron annihilates in flight, for $E_{k i n} \gg 0.5 \mathrm{MeV}$, the photon emitted in the forward direction in the laboratory system takes up nearly all of the positron's energy while the other photon has an energy of the order of a few tenths of a MeV. Given a flux of fast positrons, the energy distribution of photons produced by such fast annihilation events would be continuous in nature, and make no effective contribution to a discrete photon flux at $0.51 \mathrm{MeV}$. Heitler(14) gives the probability of annihilation in flight as a function of initial positron energy in lead, and Rocchia( 8 ) has done an approximate calculation of the same quantity in air. Assuming the energy distribution for the cosmic-ray positron flux given in Figure 1 , it is estimated from these data that approximately $15 \%$ of such positrons would annihilate in flight and thereby not contribute to the discrete gamma line at $0.51 \mathrm{MeV}$.

As positrons slow down in air, there is a reasonably high probability that they will form a bound system with an electron, known as positronium. This usually takes place at positron kinetic energies of the order of $10 \mathrm{eV}$, i.e., for $\mathrm{E}_{\text {ion }}-6.8<\mathrm{E}_{\mathrm{p}}<\mathrm{E}_{\text {ion }}(\mathrm{eV})$, where $\mathrm{E}_{\text {ion }}$ is the ionization energy for the gas atoms and $6.8 \mathrm{eV}$ is the binding, energy of positronium. One-quarter of the positronium is formed in the singlet state (parapositronium) which decays via $2 \gamma$ annihilation with a mean lifetime of $1.25 \times 10^{-10} \mathrm{sec}$. The 
remaining three-quarters is formed in the triplet state (orthopositronium) for which $2 \gamma$ annihilation is forbidden. Orthopositronium decays via $3 \gamma$ annihilation with a mean lifetime of $1.4 \times 10^{-\gamma} \mathrm{sec}$. The $2 \gamma$ annihilation of parapositronium, like that for free positrons of kinetic energies $<\mathrm{mc}^{2}$, produces only $0.51 \mathrm{MeV}$ photons, while the $3 \gamma$ annihilation produces a continuous spectrum of photons and thus makes no contribution to the $0.51 \mathrm{MeV}$ peak. However, before decaying by $3 \gamma$ emission, orthopositronium may be converted to parapositronium by various "quenching" mechanisms. In estimating the production of $0.51 \mathrm{MeV}$ photons by slow positrons, we therefore must take into account the fraction that forms positronium and the fractions of bound positrons that annihilate from the singlet and triplet states.

The probability of $3 \gamma$ annihilation from orthopositronium for low energy positrons slowing down past the ionization energy threshold is

$$
\mathrm{P}_{3 \gamma} \approx \frac{3}{4} \mathrm{~F} \frac{\lambda_{3}}{\lambda_{3}+\mathrm{p} \lambda_{\mathrm{q}}}
$$

where $F$ is the fraction of such positrons forming positronium, $p$ is the pressure in atmospheres, and $\lambda_{3}$ and $\lambda_{q}$ are the decay constants characteristic of non-quenched orthopositronium and the quenching process (per atmosphere), respectively. $\lambda_{3}$ is known to be $7.20(\mu \mathrm{sec})^{-1}$. The evaluation of $F$ and $\lambda_{q}$ is more difficult, and experimental data are not entirely concordant. The data summaries of Green and Lee(15) and Goldanskii(16) provide estimates for $F$ of $0.4-0.5$ for oxygen and 0.3 for nitrogen. For air, we choose $0.75 \mathrm{~F}=0.25$ as a reasonable estimate for the formation fraction of orthopositronium. For the partial pressures of nitrogen in the atmosphere, $\mathrm{p} \lambda_{\mathrm{q}}$ is negligible compared with $\lambda_{3}$. Thus, all quenching or orthopositronium in the atmosphere is due to the presence of oxygen, which is quite efficient in this 
regard. Osmon(17) gives $\lambda_{\mathrm{q}}>60 \mu \mathrm{sec}^{-1} \mathrm{~atm}^{-1}$ for oxygen. Assuming a value of 100, we get

$$
\begin{aligned}
\mathrm{P}_{3 \gamma} & \cong 0.07 \text { at sea-level } \\
& \cong 0.25 \text { at high altitudes. }
\end{aligned}
$$

Other measurements of $\lambda_{q}$ for oxygen indicate somewhat lower values, which would increase the sea-level value of $\mathrm{P}_{3} \gamma$ and narrow the variation of $\mathrm{P}_{3} \gamma$ with altitude. The results of celitans et $\underline{a l}^{(18)} \mathrm{give}^{(18}=22.3 \mu \mathrm{sec}_{\mathrm{q}}^{-1} \mathrm{~atm}^{-1}$, yielding $\mathrm{P}_{3 \gamma} \cong 0.15$ at sea level. Given the uncertainties associated with these data, we neglect the variation of $\mathrm{P}_{3} \gamma$ with altitude and assume that $\mathrm{P}_{3 \gamma}=0.20$ throughout the atmosphere.

We are now prepared to estimate the mean number of 0.51 $\mathrm{MeV}$ photons produced by each cosmic-ray positron at the end of its range. From the above results, the mean probability for cosmic-ray positrons to reach the ionization threshold energy is 0.85 and the probability of $2 \gamma$ annihilation below this threshold energy is 0.80 , and therefore the mean number of $0.51 \mathrm{MeV}$ photons produced per positron absorption is $2(0.85)(0.80)=1.36$.

\section{B. Relation of $0.51 \mathrm{MeV}$ Gamma Source to Positron source}

If positrons were emitted isotropically and the $s_{p}(h)$ were constant with depth, the positron absorption density would be equal to this production density, and the gamma source function would be simply

$$
\mathrm{S}_{\gamma}(\mathrm{h})=1.36 \mathrm{~s}_{\mathrm{p}}(\mathrm{h})
$$


by the result of Section III A. However, as indicated in Section II $C, S_{p}\left(h^{\prime}\right)$ varies exponentially with depth for $\left|\mathrm{h}-\mathrm{h}^{\prime}\right| \leq 20 \mathrm{gm} / \mathrm{cm}^{2}$ (the effective maximum range of the emitted positrons), where $h$ is the depth of interest for the determination of the gamma-ray function. If there is no radical change with depth in the angular distribution of emitted positrons, we may assume that the positron absorption density exhibits a variation with depth similar to that of the source density and that therefore $S_{\gamma}(h)$ shows this variation as well. However, because of this variation and the fact that positrons are preferentially emitted in the downward direction, the absorption density of positrons at a particular depth cannot be equated to the production density at that same depth. Since $|\alpha| \ll \nu$, the source variation effect is negligible, but the directional effect, though small, will introduce a systematic error into the final results.

This can be corrected for in an approximate way by considering the range spectrum of positrons given in Figure 2. For positrons emitted vertically downward, Figure 2 indicates the depth "displacement" distribution of positron annihilation from production. Taking into account the fact that actual displacement spectrum in the atmosphere will be somewhat softer than this, it can be reasonably estimated that the mean vertical displacement is 5 to $10 \mathrm{gm} / \mathrm{cm}^{2}$. We can then correlate the positron annihilation density at depth $h$ (and gamma-ray production density) with the positron production density at a slightly lesser depth, which we assume to be adequately represented by $h-8$, i.e.,

$$
s_{\gamma}(h) \cong 1.36 s_{p}(h-8)
$$

Since

$$
S_{p}(h) \cong \mathrm{Ke}^{-\alpha h}
$$

we get

$$
s_{\gamma}(h) \cong 1.36 s_{p}(h) \exp (8 \alpha)
$$




\section{Relation of $0.51 \mathrm{MeV}$ Photon Flux to Source}

Experimental data on the $0.51 \mathrm{MeV}$ photon flux in the atmosphere $(8,22)$ indicate that the depth variation of this flux can be approximated by an exponential function over depth ranges much larger than $1 / \mu$, with an attenuation length, $1 / \beta$, such that $\beta<0.1 \ll \mu$. The derivation of the relation between this flux and its source is thus quite analogous to that for the positrons (Section IIC), and a simple calculation (Appendix III) yields the expression

$$
\Phi_{\gamma}(h) \cong S_{{ }^{\prime} \gamma^{\prime}}(h) / \mu
$$

for $\mu \mathrm{h} \gg 1$ and $\beta \ll \mu$. Since $\mu \cong \nu=0.085 \mathrm{~cm}^{2} / \mathrm{gm}$, this relation holds for those depths where the analogous relation for positrons (equation II-7) is valid. 


\section{RELATION BETWEEN ELECTRON AND $0.51 \mathrm{MeV}$ PHOTON FLUXES}

IN THE ATMOSPHERE

A. Positron Flux as a Function of 0.51 MeV Photon Flux

We now collect the results of the preceding discussion. We substitute into equation III-I the equations II-7 and III-2, yielding

$$
\mu \Phi_{\gamma}(h) \cong 1.36 \nu \Phi_{p}(h) \exp (8 \alpha)
$$

or

$$
\Phi_{p}(h) \cong 0.735(\mu / \nu) e^{-8 \alpha} \Phi_{\gamma}(h)
$$

Since in our case $\mu \cong \nu=0.085 \mathrm{~cm}^{2} / \mathrm{gm}$, this can be simplified to

$$
\Phi_{\mathrm{p}}(\mathrm{h}) \cong 0.735 \mathrm{e}^{-8 \alpha} \Phi_{\gamma}(\mathrm{h})
$$

We also note that equation III-l implies that $\alpha \cong \beta$, and thus we get

$$
\Phi_{p}(h) \cong 0.735 e^{-8 \beta} \Phi_{\gamma}(h)
$$

where $\Phi_{\gamma}(h)$ and $\beta$ are experimentally determinable quantities.

\section{B. Charge Ratio of Cosmic-Ray Secondary Electrons}

The ratio of positron flux to total electron flux in the atmosphere depends on the nature of their sources, and the processes taking place within the electromagnetic cascade. Electrons produced by knock-on collisions of charged particles with atomic nuclei and compton scattering of gamma-rays con- 
sist entirely of negatrons. The production of first-generation electrons from muon decay would exhibit the $12 \%$ positive excess characteristic of the muon component. In cascade showers, a negatron excess of approximately $5 \%$ has been calculated,(19) due to the combined effects of positron annihilation, compton scattering, $\delta$-electron production, and differences in ionization loss. Beck's recent calculations(10) for photon-initiated air cascades indicate 10 - 20 percent more negatrons than positrons above $5 \mathrm{MeV}$ at depths of penetration near the transition maxima $\left(\approx 100 \mathrm{gm} / \mathrm{cm}^{2}\right)$.

With these results as a guide, we assume that the negatron flux is $10 \%$ greater than the positron flux at all altitudes, i.e.,

$$
\Phi_{\mathrm{e}}(\mathrm{h}) \cong 2.1 \Phi_{\mathrm{p}}(\mathrm{h})
$$

where $\Phi_{e}(h)$ is the total electron flux.

\section{c. Final Result}

Combining equations IV-3 and IV-4, we obtain the desired relationship between $0.51 \mathrm{MeV}$ photon flux and total electron flux, i.e.,

$$
\Phi_{e}(h) \cong 1.54 e^{-8 \beta} \Phi_{\gamma}(h)
$$

where

$$
\left.\beta=-\frac{I}{\Phi_{\gamma}(h)} \frac{d \Phi_{\gamma}(h)}{d h}\right]_{h}
$$

Thus, a measurement of $\Phi_{\gamma}(\mathrm{h})$ for all $\mathrm{h}$ is sufficient to uniquely determine the electron flux at any altitude where the assumptions involved in the derivation of equation IV-5 are valid. 
V. DETERMINATION OF ELECTRON FLUX FROM GAMMA-RAY MEASUREMENTS

The preceding discussion provides a reasonable quantitative relationship between the total electron flux and the $0.51 \mathrm{MeV}$ gamma flux within the atmosphere. Although a comparison of the electron flux inferred by this method with direct experimental measurements would be desirable, no data exist which would permit such a comparison. The omnidirectional cosmicray electron flux is reasonably well known only near the top of the atmosphere, where our method is not applicable, and near sea-level, where there are no useful $0.51 \mathrm{MeV}$ gamma-ray data. The annihilation gamma-ray flux in the atmosphere is of sufficiently low intensity that reliable measurements on balloon flights have been obtained only at atmospheric depths less than about $500 \mathrm{gm} / \mathrm{cm}^{2}$. This situation has led to the consideration of the total low-energy photon flux as a possible indicator of electron flux. If it can be shown that some measure of this flux is proportional to either the $0.51 \mathrm{MeV}$ photon or total electron flux, such a measure would be a statistically more significant experimental indicator of electron flux than the flux of $0.51 \mathrm{MeV}$ annihilation radiation.

In 1961, Peterson(12) conducted nearly simultaneous measurements of $0.51 \mathrm{MeV}$ and $37-75 \mathrm{keV}$ photon fluxes and total charged particle flux as a function of atmospheric depth at $55^{\circ} \mathrm{N}$ geomagnetic latitude. He concluded that the nearly identical depth variations of the $0.51 \mathrm{MeV}$ and 37-75 keV photon fluxes were indicative of a common origin related to the electron component. More recently, Brini et al. (13) have conducted simultaneous measurements of the photon flux in four energy bands between 40 and $190 \mathrm{keV}$ and the total charged particle flux at $45^{\circ} \mathrm{N}$ geomagnetic latitude. These authors showed that the variation with atmospheric depth of the inferred electron flux and the corresponding variation of the total counting rate for their gamma-ray detector were quite comparable, and that the inferred photon energy spectrum could be explained by assuming that the observed photons originated from electron bremsstrahlung, taking into account subsequent compton scattering and degradation. 
These data provide compelling evidence that the lowenergy end of the cosmic-ray photon spectrum in the atmosphere is mostly derived from electron bremsstrahlung. However, while the calculations of Brini et al. indicate approximate agreement between the measured and calculated photon energy spectra, it is clear that any attempt to relate the electron flux to the low energy photon flux in a more exact manner would be exceedingly complicated, and would also require the reduction of experimental data to absolute photon flux. These complications can be avoided by making use of equation IV-5 relating $0.51 \mathrm{MeV}$ photon flux to total electron flux as a means of "calibrating" any experimental measure of the low energy photon flux. This procedure is illustrated in Table $I$, utilizing Peterson's data. (12) The columns labelled $\varphi_{0.5}$, $\varphi_{\gamma}$, and $\varphi_{c p}$ are Peterson's measures of the $0.51 \mathrm{MeV}$ photon flux, total photon flux between 36 and $75 \mathrm{keV}$, and charged particle flux in units of $\mathrm{cm}^{-2} \mathrm{sec}^{-1}$. The values given for $\varphi_{e}$ are the electron fluxes calculated from $\varphi_{0.5}$ by means of equation IV-5. The ratio, $\varphi_{e} / \varphi_{\gamma}$, is seen to be approximately constant at all depths, except at 600 and $800 \mathrm{gm} / \mathrm{cm}^{2}$, where the statistics for $\varphi_{0.5}$ are very poor. The values in parentheses for $\varphi_{e}$ at these depths are those obtained by assuming $\varphi_{\mathrm{e}} / \varphi_{\gamma}=0.145$, a result inferred from the data at lesser depths. The last column indicates the inferred ratio of total electron to total charged particle flux, the parenthetical values referring to the revised values for $\varphi_{e}$. These revised values are more reasonable, since this ratio should be $\sim 0.3$ at sea-level.

The procedure to be adopted for relating any experimental measure of the low-energy gamma intensity to total electron flux follows a similar pattern. This measure $\left(\varphi_{\gamma}\right)$ is calibrated by means of $\varphi_{0.5}$ near the Pfotzer maximum. Here, $\beta \cong 0$ in equation IV -5 . We can also modify the constant factor in this equation, since $P_{3 \gamma} \cong 0.25$ at high altitudes rather than the assumed mean value of 0.20 (see Section III-A), Thus, we get

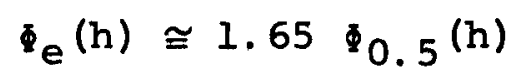

at $\mathrm{h} \cong 100 \mathrm{gm} / \mathrm{cm}^{2}$. 
We must now take into account another displacement effect analogous to that discussed in Section III-B. At lower altitudes the gamma-ray flux at any point can be regarded as being produced by electrons at slightly higher altitudes because of the generally downward orientation of the photon trajectories. Given the electron spectrum in Figure 1 and the fact that the photon production spectrum from bremsstrahlung has approximately an $\mathrm{E}^{-1}$ dependence, it is estimated that the mean photon energy at production that contributes to the observed photon flux near $100 \mathrm{keV}$ is slightly more than $1 \mathrm{MeV}$. Without analyzing this complex situation further, a qualitatively reasonable correction for this "displacement" effect can be made by assuming that the photon flux at depth $h$ can be correlated with the electron flux at depth $(h-20)$. Then the relationship between the two fluxes of interest can be approximated by

$$
\varphi_{e}(\mathrm{~h}) \cong 1.65 \varphi_{\gamma}(\mathrm{h}) \cdot \frac{\varphi_{0.5}(100)}{\varphi_{\gamma}(100)} \cdot \mathrm{e}^{-20 \beta} \quad(\mathrm{V}-1)
$$

where $B$ is the effective attenuation coefficient of the gamma-ray intensity at depth $h$ (assumed equal to that of the electrons). The factor $\left[\varphi_{0,5}(100) / \varphi_{\gamma}(100)\right]$ applicable to a particular detector can be determined on a single balloon flight and becomes a calibration factor for the experimental quantity $\varphi_{\gamma}(h)$. Not the least of the advantages of this method is the fact that $\varphi_{\gamma}(\mathrm{h})$ need not be an absolute flux, since it appears both in the numerator and the denominator of the above expression.

This procedure has been applied to the data obtained by Chupp (19) on two balloon flights from Palestine, Texas, on July 22 and November 2, 1967. On the latter flight, simultaneous determinations were made of the $0.51 \mathrm{MeV}$ gamma-ray photopeak counting rate and the total response of a $3 \times 3$ in. CsI detector surrounded by a $1 / 2$ in. charged particle shield (NE 102). The CsI detector response should be closely 
proportional to the low-energy photon flux under such conditions. Given the experimental values for the $0.51 \mathrm{MeV}$ flux and CsI response in counts $/ \mathrm{sec}$ at $\mathrm{h}=125 \mathrm{gm} / \mathrm{cm}^{3}$, the above equation $(v-1)$ was utilized to estimate the electron flux at depths $>50 \mathrm{gm} / \mathrm{cm}^{2}$. The CsI data from the July flight were then normalized to the November $2,0.5 \mathrm{MeV}$ gamma-flux value. The results for bath flights are given in Figure 3 and are seen to be quite consistent.

The validity of our procedure may be tested in an approximate way by comparing the data in Figure ${ }^{-} 3$ with estimates of the absolute electron flux in the lower atmosphere that have been obtained in the following manner:

1. An absolute value at sea level of $7 \times 10^{-3} \mathrm{~cm}^{-2} \mathrm{sec}^{-1}$ ( $\pm 10 \%$ ) inferred from Carmichael's data(4) with corrections for an arithmetic error in the reference and the more southerly geomagnetic latitude of the Texas flights.

2. The altitude profile of the response (primarily to photons) of a $4 \times 4 " \mathrm{NaI}(\mathrm{Tl})$ detector over lakes and low-background land sites between $900 \mathrm{gm} / \mathrm{cm}^{2}$ and sea-level(21), which should indicate an adequate extrapolation of the balloon data to sea-level.

3. Relative omnidirectional electron flux values inferred from the vertical flux and angular distribution data of Barker ( 6 ) obtained with a cloud chamber at Mt, Evans $\left(600 \mathrm{gm} / \mathrm{cm}^{2}\right)$ and Echo Lake $\left(690 \mathrm{gm} / \mathrm{cm}^{2}\right)$, Colorado, and Ann Arbor, Michigan.

The results of (2) and (3) are normalized to the sea-level intensity of (I). It is readily apparent that the balloon flight results in Figure 3 , the absolute values of which are determined from high-altitude data, are quite consistent with the best available information on omnidirectional electron intensities in the lower atmosphere.

The available evidence indicates that this method is probably adequate for inferring the absolute omnidirectional 
electron flux at atmospheric depths greater than $50 \mathrm{gm} / \mathrm{cm}^{2}$ to an accuracy of $\pm 10 \%$, neglecting any systematic experimental errors in the determination of absolute photon fluxes.

Multi-detector balloon packages are currently being designed at this laboratory to provide simultaneous measurements of the altitude profile of the total and $0.51 \mathrm{MeV}$ gamma-ray fluxes for use as a monitor of the secondary electron flux in the atmosphere. Results from the Monte Carlo studies of electron production and transport throughout the atmosphere should provide a basis for further refinement of the assumptions made in the present analysis, as well as the extension of this method to higher altitudes. 


\section{REFERENCES}

1. Lowder, W. M. and Beck, H. L. Cosmic Ray Ionization in the Lower Atmosphere J. Geophys. Res., 7l, 466l-4668 (1966)

2. Berger, M. J. and Seltzer, S. M.

Tables of Energy Losses and Ranges of Electrons and Positrons

NASA Report $S P=3012$ (1964)

3. Harder, D. and Poschet, G. Transmission und Reichweite schneller Elektronen in

Energiebereich 4 bis $30 \mathrm{MeV}$ Phys. Lett., 24B, 519-521 (1967).

4. Carmichael, $\mathrm{H}$.

Energy Spectrum of the Soft Component Near Sea Level Phys. Rev., 107, 1401-1409 (1957)

5. Greisen, $K$.

Intensity of Cosmic Rays at Low Altitude and the Origin of the soft component

Phys. Rev., 63, 323-333 (1943)

6. Barker, P. R.

Cosmic-Ray Electrons Near Sea-Level and at Mountain

Altitudes

Phys. Rev., 100, 860-869 (1955)

7. Charakhchian, A. M. and Charakhchian, T. N.

Measurement of Cosmic-Ray Intensity in the Stratosphere at Various Altitudes and Latitudes

Soviet Phys. JETP, 35, 761-771 (1959)

8. Rocchia, R.

Rayonnement Gamma dans I'Espace et dans I'Atmosphere Report CEA-R 2939, Saclay (1966) 
9. Butcher, J. C. and Messel, H.

Electron Number Distribution in Electron-Photon Showers in Air and Aluminum Absorbers

Nucl. Phys., 20, 15-128 (1960)

10. Beck, H. L.

Monte Carlo Calculations of the Electromagnetic Cascade in the Atmosphere (unpublished)

11. Rossi, B.

Interpretation of Cosmic-Ray Phenomena

Rev. Mod. Phys., 20, 537-583 (1948)

12. Peterson, L. E.

The 0.5 MeV Gamma-Ray and the Low-Energy Gamma-Ray Spectrum to 6 Grams per Square Centimeter over Minneapolis

J. Geophys. Res., 68, 979-987 (1963)

13. Brini, D., Ciriegi, U., Fuligni, F., and Moretti, E. Low-Energy Cosmic-Ray Photons in Atmosphere

J. Geophys. Res., 72, 903-913 (1967)

14. Heitler, W.

The Quantum Theory of Radiation

Oxford, England (1954)

15. Green, J. H. and Lee, J.

Positronium Chemistry

Academic Press, New York (1964)

16. Goldanskii, V. I.

Physical Chemistry of the Positron and Positronium

Atomic Energy Review (IAEA), 6 , 3-148 (1968).

17. Osmon, P. E.

Positron Lifetime Spectra in Molecular Gases

Phys. Rev., 140, A8-AIl (3965) 
18. Celitans, G. J., Tao, S. J., and Green, J. H. Formation and Quenching of Positronium in Gases, II. Results Obtained with the positron Lifetime Method Proc. Phys. Soc., 83, 833-842 (1964)

19. Guzhavin, V. V., Ivanenko, I. P., and Levitin, A. E. On the Electron Excess in Electromagnetic Cascade Showers

Canad. J. Phys., 46, (Pt. 2), 5209-5211 (1968)

20. Chupp, E. L., University of New Hampshire Personal Communication (1968)

21. Beck, H. L., Lowder, W. M., Bennett, B. G., and Condon, W. J. Further Studies of External Environmental Radiation USAEC Report HASL-170 (1966) 
TABLE I

EXPERIMENTAL AND INFERRED PHOTON AND PARTICLE FLUXES*

\begin{tabular}{ccccccc}
\hline $\begin{array}{c}\mathrm{h} \\
\left(\mathrm{gm} / \mathrm{cm}^{2}\right)\end{array}$ & $\varphi_{0.5}$ & $\varphi_{\gamma}$ & $\varphi_{\mathrm{cp}}$ & $\varphi_{\mathrm{e}}$ & $\varphi_{\mathrm{e}} / \varphi_{\gamma}$ & $\varphi_{\mathrm{e}} / \varphi_{\mathrm{cp}}$ \\
\hline 50 & 0.51 & 5.2 & 2.2 & 0.79 & 0.15 & 0.34 \\
100 & 0.57 & 6.0 & 2.0 & 0.88 & 0.15 & 0.38 \\
150 & 0.51 & 5.1 & 1.4 & 0.77 & 0.15 & 0.54 \\
200 & 0.40 & 4.2 & 1.05 & 0.59 & 0.14 & 0.56 \\
300 & 0.22 & 2.5 & 0.56 & 0.32 & 0.13 & 0.57 \\
400 & 0.14 & 1.4 & 0.31 & 0.21 & 0.15 & 0.68 \\
500 & 0.08 & 0.85 & 0.17 & 0.12 & 0.14 & 0.71 \\
600 & $\sim 0.05$ & 0.42 & 0.09 & $0.07(0.06)$ & 0.17 & $0.78(0.67)$ \\
800 & $\sim 0.017$ & 0.14 & $\sim 0.04$ & $0.025(0.020)$ & 0.18 & $0.63(0.50)$ \\
& & & & & & \\
\hline
\end{tabular}

*Symbols defined in text (Section V). 
APPENDIX I

CALCULATION OF POSITRON ENERGY SPECTRUM AT PRODUCTION

In Section II A, we derive a positron range spectrum from the sea-level energy spectrum of the total electron flux, arguing that the latter spectrum should be representative of the positron flux throughout most of the atmosphere. The shape of the range spectrum of the positron source is then derived from the flux range spectrum by a mathematical artifice that depends on the fortunate exponential shape of the latter.

An independent test of the derived range spectrum at production is provided by calculations recently carried out by H. L. Beck of this laboratory (10). He has calculated the properties of electromagnetic cascades in air initiated by negatrons, positrons, and photons of various energies by means of a Monte carlo code similar to that of Butcher and Messel, $(\theta)$ but improved to the extent that it uses more recent cross-sections, treats negatrons and positrons separately, and takes into account the energy dependence of the radiative cross-section of electrons in determining the distances between electron interactions and also the effect of positron annihilation in flight. The energy and the air thickness from the origin at production was scored for each secondary positron created with kinetic energy $>\mathrm{I} \mathrm{MeV}$, and integral energy spectra obtained for ten thickness intervals between 0 and $400 \mathrm{gm} / \mathrm{cm}^{2}$. The total positron production spectra at various atmospheric depths for showers initiated by electrons or photons of $0.2,0.5$, 1.0 , and $2.0 \mathrm{GeV}$ were obtained by assuming a flux of initiating particles of each energy that was constant with depth, i.e., by essentially averaging the integral energy spectra at production over all depths $<400 \mathrm{gm} / \mathrm{cm}^{2}$. The results for electron - and phocon - initiated cascades were averaged to obtain an appropriate mean value for each initiating energy. 
These integral energy spectra at the four input energies were nearly identical for energies below $100 \mathrm{MeV}$. The average values for the four energies are plotted in Figure 1 . It is quite evident that the shape of the positron energy distribution at production from such cascades is essentially the same as the shape of the sea-level energy distribution of the electron flux. The range spectrum of these positrons would then be that given in Figure 2. This fact supports the results of section II to the extent that the bulk of positrons produced in the atmosphere can be considered to derive from cascade showers initiated by electrons and photons of energies of the order of $1 \mathrm{GeV}$. 


\begin{abstract}
APPENDIX II
CALCULATION OF POSITRON FLUX IN THE ATMOSPHERE
\end{abstract}

We define

$\mathrm{R}$, positron Iinear range in $\mathrm{gm} / \mathrm{cm}^{3}$, i.e., the minimum air thickness between points of production and annihilation,

h, atmospheric depth, $\mathrm{gm} / \mathrm{cm}^{3}$,

$S_{\mathrm{p}}^{\circ}(\mathrm{R}, \mathrm{h})$, positron differential range spectrum at depth, $h$, in units of positrons $\mathrm{gm}^{-1} \mathrm{sec}^{-1} \mathrm{gm}^{-1} \mathrm{~cm}^{2}$,

$S_{p}(h)$, positron source function in units of positrons $\mathrm{gm}^{-2}$ $\sec ^{-1}\left[=\int_{0}^{\infty} S_{p}(R, h) d R\right]$, $\varphi_{p}(R, h)$, positron differential flux in units of $\mathrm{cm}^{-2} \mathrm{sec}^{-1}$
$\mathrm{gm}^{-1} \mathrm{~cm}^{2}$

$\Phi_{\mathrm{p}}(\mathrm{h})$, positron $f l u x$ in units of $\mathrm{cm}^{-2} \sec ^{-1}\left[=\int_{0}^{\infty} \varphi_{\mathrm{p}}(\mathrm{R}, \mathrm{h}) \mathrm{dR}\right]$,

p (h), air density at depth, $h$,

$t$, air thickness in $\mathrm{gm} / \mathrm{cm}^{3}$ between source and detector.

Case 1 : Positrons of all energies emitted isotropically in the laboratory system.

The basic equation is

$$
\Phi_{p}(h)=\int \frac{\rho\left(h^{\prime}\right)}{4 \pi r^{2}}\left[\int_{t}^{\infty} S_{p}\left(R, h^{\prime}\right) d R\right] d V
$$


To solve this equation, we introduce the result discussed in Section II A (equation II-5), i.e.,

$$
S_{p}(R, h) \cong \nu S_{p}^{\circ}(h) e^{-\nu R}
$$

Substituting (2) into (1), we get

$$
\begin{aligned}
\Phi_{p}(h) & =\int \frac{\rho\left(h^{\prime}\right)}{4 \pi r^{a}} S_{p}^{o}\left(h^{\prime}\right) e^{-\nu t} d v \\
& =\iiint \rho\left(h^{\prime}\right) s_{p}^{o}\left(h^{\prime}\right) e^{-\nu t} \sin \theta d \theta d r
\end{aligned}
$$

For the upper hemisphere,

$$
\begin{aligned}
d h^{\prime} & =-\rho\left(h^{\prime}\right) d z \\
d r & =\sec \theta d z=-\frac{\sec \theta}{\rho\left(h^{\prime}\right)} d h^{\prime} \\
d t & =\rho\left(h^{\prime}\right) d r=-\sec \theta d h^{\prime} \\
t & =\sec \theta\left(h-h^{\prime}\right)
\end{aligned}
$$

Let

$$
\begin{aligned}
p & \equiv \nu\left(h-h^{\prime}\right) \sec \theta \\
d_{p} & =p \sin \theta \sec \theta d \theta
\end{aligned}
$$

Then, equation (3b) becomes 


$$
\begin{aligned}
\Phi_{p}^{u}(h) & =\frac{1}{2} \int_{0}^{h} S_{p}^{o}\left(h^{\prime}\right) d h^{\prime} \int_{\nu\left(h-h^{\prime}\right)}^{\infty} \frac{e^{-p}}{p} d p \\
& =\frac{1}{2} \int_{0}^{h} S_{p}^{0}\left(h^{\prime}\right) E_{2}\left[\nu\left(h-h^{\prime}\right)\right] d h^{\prime}
\end{aligned}
$$

For the lower hemisphere, a similar integration yields

$$
\Phi_{p}^{l}(h)=\frac{1}{2} \int_{h}^{\infty} S_{p}^{\circ}\left(h^{\prime}\right) E_{1}\left[\nu\left(h^{\prime}-h\right)\right] d h^{\prime}
$$

The total flux is then

$$
\Phi_{p}(h)=\Phi_{p}^{u}(h)+\Phi_{p}^{l}(h)=\frac{1}{2} \int_{0}^{\infty} S_{p}^{o}\left(h^{\prime}\right) E_{q}\left[\nu / h-h^{\prime} \mid\right] d h^{\prime}
$$

We now assume that, over a range of $\left(h-h^{\prime}\right)$ where the integrand in (4) significantly contributes to the integral, the following relation holds

$$
\mathrm{S}_{\mathrm{p}}^{\circ}\left(\mathrm{h}^{\prime}\right) \cong \mathrm{Ke} \mathrm{e}^{-\alpha \mathrm{h}^{\prime}}
$$

Inserting (5) into (4) and integrating, we get

$$
\begin{aligned}
\Phi_{\mathrm{p}}(\mathrm{h}) & =\frac{K \mathrm{e}^{-\alpha h}}{2 \alpha} \text { ln } \frac{1+\alpha / \nu}{1-\alpha / \nu}+\frac{K}{2 \alpha}\left[E_{1}(\nu \mathrm{h})-e^{-\alpha h} E_{1}(\nu h-\alpha h)\right] \\
& \cong \frac{K e^{-\alpha h}}{\nu} \text { for } \nu \mathrm{h} \gg 1, \alpha \ll \nu
\end{aligned}
$$


Case 2: Positrons emitted in downward direction.

In this case, the equivalent of equation ( 1 ) is

$$
{ }_{p}(h)=\int \rho\left(h^{\prime}\right)\left[\int_{t}^{\infty} S_{p}\left(R, h^{\prime}\right) d R\right] d V
$$

Using cylindrical coordinates and equation (2), this is readily reducible to

$$
\begin{aligned}
\Phi_{p}(h) & =\int_{0}^{h} S_{p}^{o}\left(h^{\prime}\right) e^{-\nu\left(h-h^{\prime}\right)} d\left(h-h^{\prime}\right) \\
& =K e^{-\nu h} \int_{0}^{h} e^{(\nu-\alpha) h^{\prime}} d h^{\prime} \\
& =\frac{K}{\nu-\alpha}\left(e^{-\alpha h}-e^{-\nu h}\right)
\end{aligned}
$$

For $\nu \gg \alpha$ and $\nu$ h $<1$, equation (7) is essentially identical to $(6 \mathrm{~b})$, indicating that the calculated fluxes are insensitive to the details of the angular distribution of the emitted positrons. 


\section{APPENDIX III}

\section{CALCULATION OF $0.51 \mathrm{MEV}$ GAMMA-RAY FLUX IN THE ATMOSPHERE}

Utilizing the same definitions as in Appendix II, the basic equation is

$$
\Phi_{\gamma}(h)=\int \frac{\rho\left(h^{\prime}\right)}{4 \pi r^{2}} s_{\gamma}\left(h^{\prime}\right) e^{-\mu t} d V
$$

This is of the same form as equation (3a) in Appendix II, and therefore we get (see equation (6a) in Appendix II)

$$
\Phi_{\gamma}(h)=\frac{1}{2} \int_{0}^{\infty} s_{\gamma}\left(h^{\prime}\right) E_{1}\left[\mu\left|h-h^{\prime}\right|\right] d h^{\prime}
$$

Again as in Appendix II, we assume that, over a range of ( $h-h^{\prime}$ ) where the integrand significantly contributes to the integral, the following relation holds

$$
S_{\gamma}\left(h^{\prime}\right) \cong c e^{-\beta h^{\prime}}
$$

which yields

$$
\begin{aligned}
\Phi_{\gamma}(h) & =\frac{C e^{-\beta h}}{2 \beta} \text { in } \frac{1+\beta / \mu}{1-\beta / \mu}+\frac{C}{2 \beta}\left[E_{g}(\mu h)-e^{-\beta h} E_{1}(\mu h-\beta h)\right] \\
& =\frac{C e^{-\beta h}}{\mu} \text { for } \mu h \gg 1, \beta \ll \mu
\end{aligned}
$$

These relations are of the same form as (6a) and (6b) of Appendix II. 


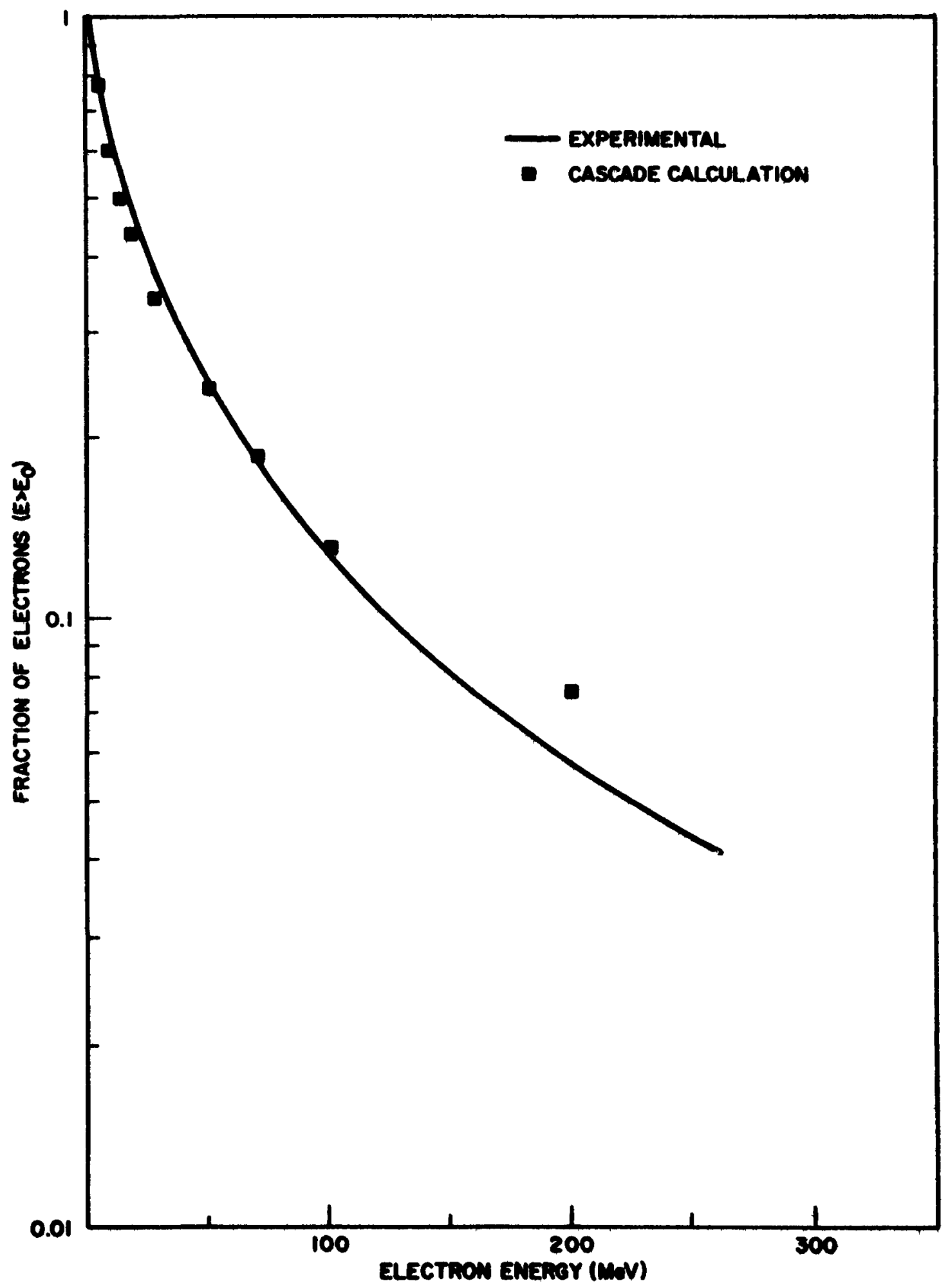

Figure 1. Integral electron energy spectrum in the atmosphere $\left(\mathrm{h}>70 \mathrm{gm} / \mathrm{cm}^{2}\right)$. 


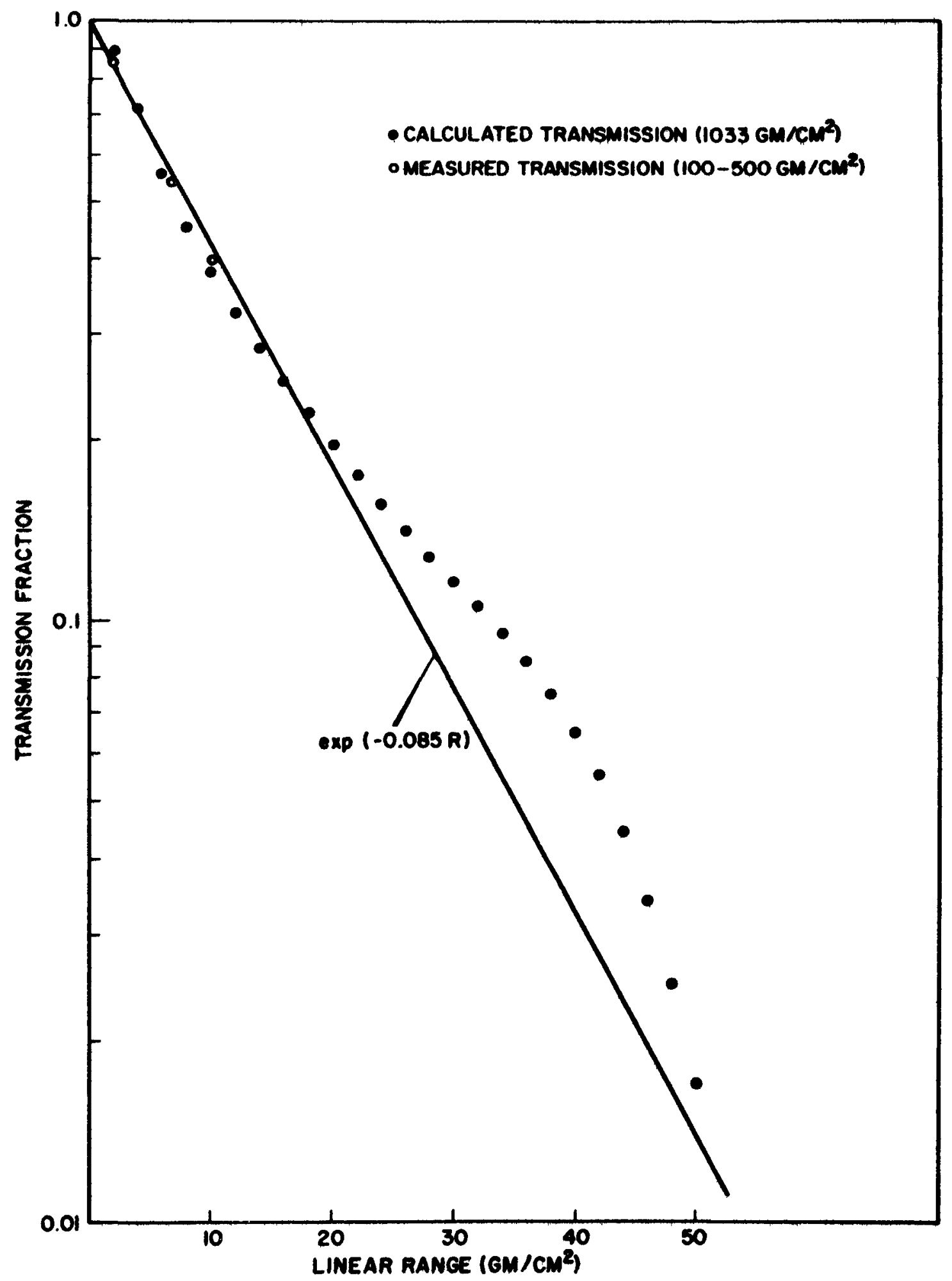

Figure 2. Integral positron range spectrum in the atmosphere $\left(\mathrm{h}>70 \mathrm{gm} / \mathrm{cm}^{2}\right)$. 


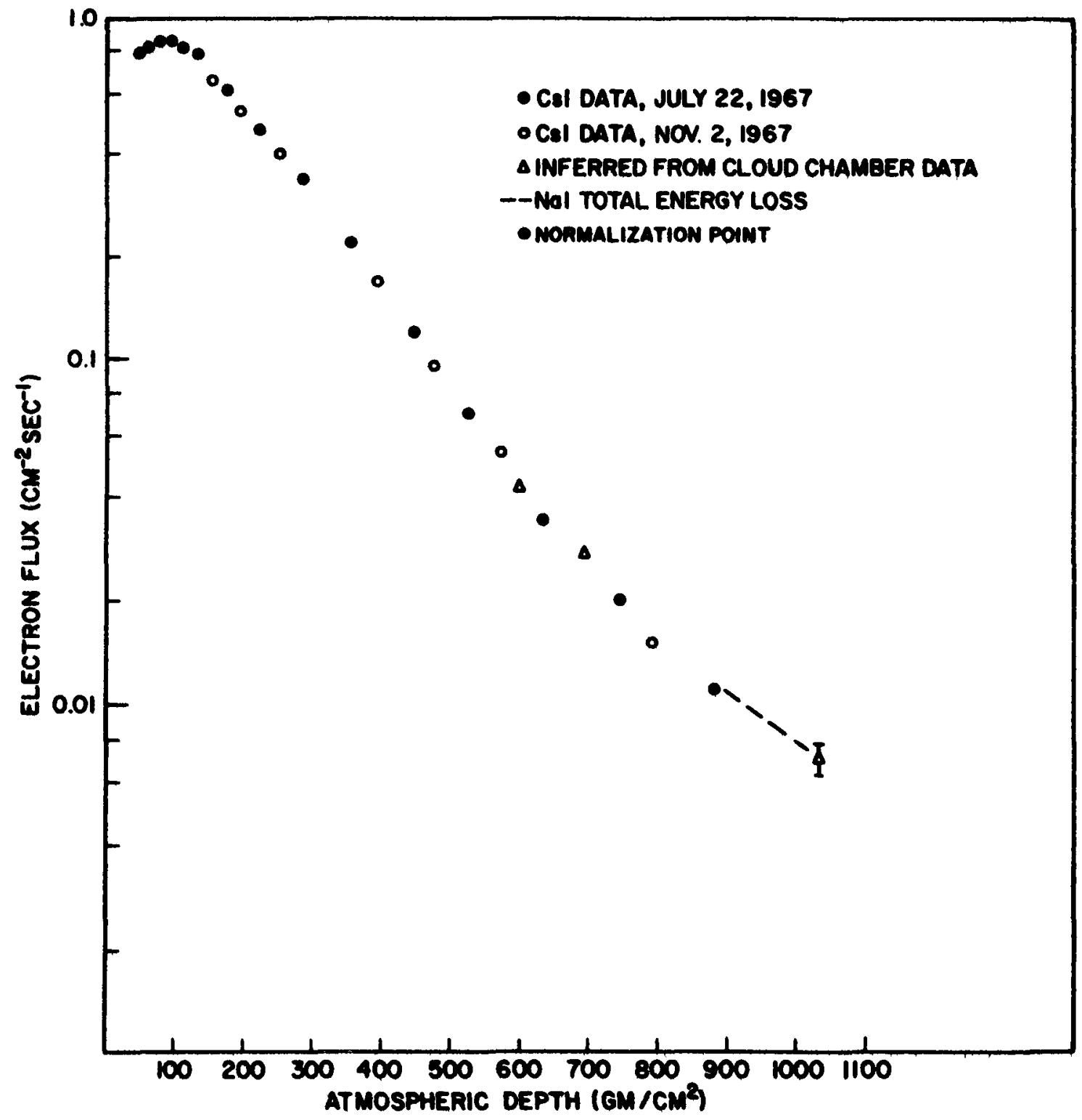

Figure 3. Secondary electron flux vs. atmospheric depth, Palestine, Texas, 1967. 